\title{
Local Fractional Fourier Series with Application to Wave Equation in Fractal Vibrating String
}

\author{
Ming-Sheng Hu, ${ }^{1}$ Ravi P. Agarwal, ${ }^{2}$ and Xiao-Jun Yang ${ }^{3}$ \\ ${ }^{1}$ Institute of Software Science, Zhengzhou Normal University, Zhengzhou 450044, China \\ ${ }^{2}$ Department of Mathematics, Texas A and M University, Kingsville, TX 78363-8202, USA \\ ${ }^{3}$ Department of Mathematics and Mechanics, China University of Mining and Technology, Jiangsu, \\ Xuzhou 221008, China \\ Correspondence should be addressed to Xiao-Jun Yang, dyangxiaojun@163.com
}

Received 14 August 2012; Revised 25 October 2012; Accepted 8 November 2012

Academic Editor: Lan Xu

Copyright (c) 2012 Ming-Sheng Hu et al. This is an open access article distributed under the Creative Commons Attribution License, which permits unrestricted use, distribution, and reproduction in any medium, provided the original work is properly cited.

\begin{abstract}
We introduce the wave equation in fractal vibrating string in the framework of the local fractional calculus. Our particular attention is devoted to the technique of the local fractional Fourier series for processing these local fractional differential operators in a way accessible to applied scientists. By applying this technique we derive the local fractional Fourier series solution of the local fractional wave equation in fractal vibrating string and show the fundamental role of the MittagLeffler function.
\end{abstract}

\section{Introduction}

Fractional calculus arises in many problems of physics, continuum mechanics, viscoelasticity, and quantum mechanics, and other branches of applied mathematics and nonlinear dynamics have been studied [1-7]. In general, the fractional analogues are obtained by changing the classical time derivative by a fractional one, which can be Riemann-Liouville, Caputo, or another one. Many classical partial differential equations possess a fractional analogue, like the fractional diffusion-wave equation [8-12], the fractional diffusion equation $[13-16]$, the fractional wave equation $[17,18]$, the fractional Schrödinger equation $[19,20]$, the fractional heat equation [21], the fractional KdV equation [22], the fractional FokkerPlanck equations [23], the fractional Fick's law [24], the fractional evolution equation [25], the Fractional Heisenberg equation [26], the fractional Ginzburg-Landau equation [27], Fractional hydrodynamic equation [28], the fractional seepage flow equation [29], and the fractional KdV-Burgers equation [30]. 
There also are other methods for solving fractional differential equations, for example, the fractional variational iteration method [31, 32] and the fractional complex transform [33-37]. In all of the methods mentioned above, the solutions of the fractional differential equations should be analytical if the fractional derivative is in the Caputo or RiemannLiouville sense. However, some solutions to ordinary and partial differential equations are fractal curves. As a result, we cannot employ the classical Fourier series, which requires that the defined functions should be differentiable, to describe some solutions to ordinary and partial differential equations in fractal space. However, based on the modified RiemannLiouville derivative, Jumarie structured a Jumurie's calculus of fractional order [38] (which is one of useful tools to deal with everywhere continuous but nowhere differentiable functions) and its applications were taken into account in Probability calculus of fractional order [39], Laplace transform of fractional order via the Mittag-Leffler function (in convenient Hilbert space) [40], and adomian decomposition method for nonsmooth initial value problems [41]. Local fractional calculus is revealed as one of useful tools to deal with everywhere continuous but nowhere differentiable functions in areas ranging from fundamental science to engineering [42-57]. For these merits, local fractional calculus was successfully applied in the local fractional Laplace problems [53, 54], local fractional Fourier analysis [53, 54], local fractional short time transform [53, 54], local fractional wavelet transform [53-55], fractal signal [55, 56], and local fractional variational calculus [57].

In this paper we introduce a local fractional wave equation in fractal vibrating string which is described as

$$
\frac{\partial^{2 \alpha} u(x, t)}{\partial t^{2 \alpha}}+a^{2 \alpha} \frac{\partial^{2 \alpha} u(x, t)}{\partial x^{2 \alpha}}=0
$$

with fractal boundary conditions

$$
\begin{gathered}
u(0, t)=u(l, t)=0, \\
u(0, t)=\frac{\partial^{\alpha} u(l, t)}{\partial x^{\alpha}}=0, \\
u(x, 0)=f(x), \\
\frac{\partial^{\alpha} u(l, 0)}{\partial x^{\alpha}}=g(x),
\end{gathered}
$$

where $\partial^{2 \alpha} u(x, t) / \partial t^{2 \alpha}, \partial^{2 \alpha} u(x, t) / \partial x^{2 \alpha}, \partial^{\alpha} u(l, 0) / \partial x^{\alpha}$, and $\partial^{\alpha} u(l, t) / \partial x^{\alpha}$ are local fractional partial differential operator, and where $u(x, t)$ is local fractional continuous (for more details, see $[53,54])$. We study the technique of the local fractional Fourier series for treating the local fractional wave equation in fractal vibrating string. This paper is organized as follows. In Section 2, we specify and investigate the concepts of local fractional calculus and local fractional Fourier series. In Section 3, we present the solving process for local fractional wave equation with local fractional derivative. In Section 4, we study the expression solution with Mittag-Leffler functions in fractal space. Finally, Section 5 is conclusions. 


\section{Preliminaries}

In this section we start with local fractional continuity of functions, and we introduce the notions of local fractional calculus and local fractional Fourier series.

\subsection{Local Fractional Continuity of Functions}

In order to discuss the local fractional continuity of nondifferential functions on fractal sets, we first consider the following results.

Lemma 2.1 (see [57]). Let $F$ be a subset of the real line and be a fractal. If $f:(F, d) \rightarrow\left(\Omega^{\prime}, d^{\prime}\right)$ is a bi-Lipschitz mapping, then there are for constants $\rho, \tau>0$, and $F \subset R$,

$$
\rho^{s} H^{s}(F) \leq H^{s}(f(F)) \leq \tau^{s} H^{s}(F),
$$

such that for all $x_{1}, x_{2} \in F$,

$$
\rho^{\alpha}\left|x_{1}-x_{2}\right|^{\alpha} \leq\left|f\left(x_{1}\right)-f\left(x_{2}\right)\right| \leq \tau^{\alpha}\left|x_{1}-x_{2}\right|^{\alpha} .
$$

As a direct result of Lemma 2.1, we have, [57],

$$
\left|f\left(x_{1}\right)-f\left(x_{2}\right)\right| \leq \tau^{\alpha}\left|x_{1}-x_{2}\right|^{\alpha},
$$

such that

$$
\left|f\left(x_{1}\right)-f\left(x_{2}\right)\right|<\varepsilon^{\alpha},
$$

where $\alpha$ is fractal dimension of $F$. The result that is directly deduced from fractal geometry is related to fractal coarse-grained mass function $\gamma^{\alpha}[F, a, b]$, which reads, [57],

$$
\gamma^{\alpha}[F, a, b]=\frac{H^{\alpha}(F \cap(a, b))}{\Gamma(1+\alpha)}
$$

with

$$
H^{\alpha}(F \cap(a, b))=(b-a)^{\alpha},
$$

where $H^{\alpha}$ is $\alpha$ dimensional Hausdorff measure.

Notice that we consider the dimensions of any fractal spaces (e.g., Cantor spaces or like-Cantor spaces) as a positive number. It looks like Euclidean space because its dimension is also a positive number. The detailed results had been considered in [53, 54,57].

Definition 2.2. If there exists, $[53,57]$,

$$
\left|f(x)-f\left(x_{0}\right)\right|<\varepsilon^{\alpha},
$$


with $\left|x-x_{0}\right|<\delta$, for $\varepsilon, \delta>0$ and $\varepsilon, \delta \in R$, then $f(x)$ is called local fractional continuous at $x=x_{0}$, denoted by $\lim _{x \rightarrow x_{0}} f(x)=f\left(x_{0}\right) . f(x)$ is called local fractional continuous on the interval $(a, b)$, denoted by

$$
f(x) \in C_{\alpha}(a, b),
$$

if (2.7) is valid for $x \in(a, b)$.

Definition 2.3. If a function $f(x)$ is called a nondifferentiable function of exponent $\alpha, 0<\alpha \leq 1$, which satisfies Hölder function of exponent $\alpha$, then for $x, y \in X$ such that, [54, 57],

$$
|f(x)-f(y)| \leq C|x-y|^{\alpha}
$$

Definition 2.4. A function $f(x)$ is called to be continuous of order $\alpha, 0<\alpha \leq 1$, or shortly $\alpha$ continuous, when we have that, [54,57],

$$
f(x)-f\left(x_{0}\right)=o\left(\left(x-x_{0}\right)^{\alpha}\right)
$$

Remark 2.5. Compared with (2.10), (2.7) is standard definition of local fractional continuity. Here (2.9) is unified local fractional continuity [57].

\subsection{Local Fractional Derivatives and Integrals}

Definition 2.6 (let $f(x) \in C_{\alpha}(a, b)$ ). Local fractional derivative of $f(x)$ of order $\alpha$ at $x=x_{0}$ is given, [53-57],

$$
f^{(\alpha)}\left(x_{0}\right)=\left.\frac{d^{\alpha} f(x)}{d x^{\alpha}}\right|_{x=x_{0}}=\lim _{x \rightarrow x_{0}} \frac{\Delta^{\alpha}\left(f(x)-f\left(x_{0}\right)\right)}{\left(x-x_{0}\right)^{\alpha}},
$$

where $\Delta^{\alpha}\left(f(x)-f\left(x_{0}\right)\right) \cong \Gamma(1+\alpha) \Delta\left(f(x)-f\left(x_{0}\right)\right)$.

For any $x \in(a, b)$, there exists, [53-57],

$$
f^{(\alpha)}(x)=D_{x}^{(\alpha)} f(x)
$$

denoted by

$$
f(x) \in D_{x}^{(\alpha)}(a, b)
$$

Local fractional derivative of high order is derived as, [57],

$$
f^{(k \alpha)}(x)=\overbrace{D_{x}^{(\alpha)} \cdots D_{x}^{(\alpha)}}^{k \text { times }} f(x),
$$


and local fractional partial derivative of high order, [57],

$$
\frac{\partial^{k \alpha} f(x)}{\partial x^{k \alpha}}=\overbrace{\frac{\partial^{\alpha}}{\partial x^{\alpha}} \cdots \frac{\partial^{\alpha}}{\partial x^{\alpha}}}^{k \text { times }} f(x)
$$

Definition 2.7 (let $f(x) \in C_{\alpha}(a, b)$ ). Local fractional integral of $f(x)$ of order $\alpha$ in the interval $[a, b]$ is given by, [53-57],

$$
{ }_{a} I_{b}^{(\alpha)} f(x)=\frac{1}{\Gamma(1+\alpha)} \int_{a}^{b} f(t)(d t)^{\alpha}=\frac{1}{\Gamma(1+\alpha)} \lim _{\Delta t \rightarrow 0} \sum_{j=0}^{j=N-1} f\left(t_{j}\right)\left(\Delta t_{j}\right)^{\alpha}
$$

where $\Delta t_{j}=t_{j+1}-t_{j}, \Delta t=\max \left\{\Delta t_{1}, \Delta t_{2}, \Delta t_{j}, \ldots\right\}$, and $\left[t_{j}, t_{j+1}\right], j=0, \ldots, N-1, t_{0}=a, t_{N}=b$, is a partition of the interval $[a, b]$.

For convenience, we assume that

$$
{ }_{a} I_{a}^{(\alpha)} f(x)=0 \quad \text { if } a=b, \quad{ }_{a} I_{b}^{(\alpha)} f(x)={ }_{b} I_{a}^{(\alpha)} f(x) \quad \text { if } a<b .
$$

For any $x \in(a, b)$, we get, [53, 54, 57],

$$
{ }_{a} I_{x}^{(\alpha)} f(x),
$$

denoted by

$$
f(x) \in I_{x}^{(\alpha)}(a, b)
$$

Remark 2.8. If $f(x) \in D_{x}^{(\alpha)}(a, b)$, or $I_{x}^{(\alpha)}(a, b)$, we have that, $[46,47,50]$,

$$
f(x) \in C_{\alpha}(a, b) .
$$

\subsection{Special Functions in Fractal Space}

Definition 2.9. The Mittag-Leffler function in fractal space is defined by, [53, 57],

$$
E_{\alpha}\left(x^{\alpha}\right):=\sum_{k=0}^{\infty} \frac{x^{\alpha k}}{\Gamma(1+k \alpha)}, \quad x \in R, 0<\alpha \leq 1
$$

Definition 2.10. The sine function in fractal space is given by the expression, [54, 57],

$$
\sin _{\alpha} x^{a}:=\sum_{k=0}^{\infty}(-1)^{k} \frac{x^{\alpha(2 k+1)}}{\Gamma[1+\alpha(2 k+1)]}, \quad x \in R, 0<\alpha \leq 1 .
$$


Definition 2.11. The cosine function in fractal space is given, [54, 57],

$$
\cos _{\alpha} x^{a}:=\sum_{k=0}^{\infty}(-1)^{k} \frac{x^{2 \alpha k}}{\Gamma(1+2 \alpha k)}, \quad x \in R, 0<\alpha \leq 1 .
$$

The following rules hold [54,57]:

$$
\begin{aligned}
& E_{\alpha}\left(x^{\alpha}\right) E_{\alpha}\left(y^{\alpha}\right)=E_{\alpha}\left((x+y)^{\alpha}\right), \quad E_{\alpha}\left(x^{\alpha}\right) E_{\alpha}\left(-y^{\alpha}\right)=E_{\alpha}\left((x-y)^{\alpha}\right), \\
& E_{\alpha}\left(i^{\alpha} x^{\alpha}\right) E_{\alpha}\left(i^{\alpha} y^{\alpha}\right)=E_{\alpha}\left(i^{\alpha}(x+y)^{\alpha}\right), \quad E_{\alpha}\left(i^{\alpha} x^{\alpha}\right)=\cos _{\alpha} x^{\alpha}+i^{\alpha} \sin _{\alpha} x^{\alpha}, \\
& \sin _{\alpha} x^{\alpha}=\frac{E_{\alpha}\left(i^{\alpha} x^{\alpha}\right)-E_{\alpha}\left(-i^{\alpha} x^{\alpha}\right)}{2 i^{\alpha}}, \quad \cos _{\alpha} x^{\alpha}=\frac{E_{\alpha}\left(i^{\alpha} x^{\alpha}\right)+E_{\alpha}\left(-i^{\alpha} x^{\alpha}\right)}{2}, \\
& \cos _{\alpha}(-x)^{\alpha}=\cos _{\alpha} x^{\alpha}, \quad \sin _{\alpha}(-x)^{\alpha}=-\sin _{\alpha} x^{\alpha}, \\
& \cos _{\alpha}^{2} x^{\alpha}+\sin _{\alpha}^{2} x^{\alpha}=1, \quad \sin _{\alpha}^{2} x^{\alpha}=\frac{1-\cos _{\alpha}(2 x)^{\alpha}}{2}, \\
& \cos _{\alpha}^{2} x^{\alpha}=\frac{1+\cos _{\alpha}(2 x)^{\alpha}}{2}, \quad \tan _{\alpha} x^{\alpha}=\frac{\sin _{\alpha}(2 x)^{\alpha}}{1+\cos _{\alpha}(2 x)^{\alpha}}=\frac{1-\cos _{\alpha}(2 x)^{\alpha}}{\sin _{\alpha}(2 x)^{\alpha}}, \\
& \sin _{\alpha}(2 x)^{\alpha}=2 \sin _{\alpha} x^{\alpha} \cos _{\alpha} x^{\alpha}, \quad \cos _{\alpha}(2 x)^{\alpha}=\cos _{\alpha}^{2} x^{\alpha}-\sin _{\alpha}^{2} x^{\alpha}, \\
& \tan _{\alpha}(2 y)^{\alpha}=\frac{2 \tan _{\alpha} y^{\alpha}}{1+\tan _{\alpha}^{2} x^{\alpha}}, \quad \sin _{\alpha}(2 x)^{\alpha}=\frac{2 \tan _{\alpha} x^{\alpha}}{1+\tan _{\alpha}^{2} x^{\alpha}}, \\
& \cos _{\alpha}(2 x)^{\alpha}=\frac{1-\tan _{\alpha}^{2} x^{\alpha}}{1+\tan _{\alpha}^{2} x^{\alpha}}, \quad \tan _{\alpha}(x+y)^{\alpha}=\frac{\tan _{\alpha} x^{\alpha}+\tan _{\alpha} y^{\alpha}}{1+\tan _{\alpha} x^{\alpha} \tan _{\alpha} y^{\alpha}}, \\
& \cos _{\alpha} x^{\alpha}+\cos _{\alpha} y^{\alpha}=2 \cos _{\alpha}\left(\frac{x+y}{2}\right)^{\alpha} \cos _{\alpha}\left(\frac{x-y}{2}\right)^{\alpha} \text {, } \\
& \cos _{\alpha} x^{\alpha}-\cos _{\alpha} y^{\alpha}=-2 \sin _{\alpha}\left(\frac{x+y}{2}\right)^{\alpha} \sin _{\alpha}\left(\frac{x-y}{2}\right)^{\alpha}, \\
& \sin _{\alpha} x^{\alpha}+\sin _{\alpha} y^{\alpha}=2 \sin _{\alpha}\left(\frac{x+y}{2}\right)^{\alpha} \cos _{\alpha}\left(\frac{x-y}{2}\right)^{\alpha} \text {, } \\
& \sin _{\alpha} x^{\alpha}-\sin _{\alpha} y^{\alpha}=2 \cos _{\alpha}\left(\frac{x+y}{2}\right)^{\alpha} \sin _{\alpha}\left(\frac{x-y}{2}\right)^{\alpha}, \\
& \cos _{\alpha}(x+y)^{\alpha}=\cos _{\alpha} x^{\alpha} \cos _{\alpha} y^{\alpha}-\sin _{\alpha} x^{\alpha} \sin _{\alpha} y^{\alpha}, \\
& \cos _{\alpha}(x-y)^{\alpha}=\cos _{\alpha} x^{\alpha} \cos _{\alpha} y^{\alpha}+\sin _{\alpha} x^{\alpha} \sin _{\alpha} y^{\alpha}, \\
& \sin _{\alpha}(x+y)^{\alpha}=\sin _{\alpha} x^{\alpha} \cos _{\alpha} y^{\alpha}+\cos _{\alpha} x^{\alpha} \sin _{\alpha} y^{\alpha}, \\
& \cos _{\alpha} x^{\alpha} \cos _{\alpha} y^{\alpha}=\frac{\cos _{\alpha}(x+y)^{\alpha}+\cos _{\alpha}(x-y)^{\alpha}}{2},
\end{aligned}
$$


Abstract and Applied Analysis

$$
\begin{aligned}
& \sin _{\alpha} x^{\alpha} \sin _{\alpha} y^{\alpha}=-\frac{\cos _{\alpha}(x+y)^{\alpha}-\cos _{\alpha}(x-y)^{\alpha}}{2}, \\
& \sin _{\alpha} x^{\alpha} \cos _{\alpha} y^{\alpha}=\frac{\sin _{\alpha}(x+y)^{\alpha}+\sin _{\alpha}(x-y)^{\alpha}}{2}, \\
& \sin _{\alpha}(m x)^{\alpha} \sin _{\alpha}(n x)^{\alpha}=\frac{\cos _{\alpha}((m-n) x)^{\alpha}-\cos _{\alpha}((m+n) x)^{\alpha}}{2}, \\
& \cos _{\alpha}(n x)^{\alpha} \sin _{\alpha}(m x)^{\alpha}=\frac{\sin _{\alpha}((m+n) x)^{\alpha}-\sin _{\alpha}((m-n) x)^{\alpha}}{2}, \\
& E_{\alpha}\left(i^{\alpha}(n x)^{\alpha}\right)=\left(\cos _{\alpha}(n x)^{\alpha}+i^{\alpha} \sin _{\alpha}(n x)^{\alpha}\right)^{n}, \\
& \sum_{k=1}^{n} \sin _{\alpha}(n x)^{\alpha}=\frac{\sin _{\alpha}(n x / 2)^{\alpha}}{\sin _{\alpha}(x / 2)^{\alpha}} \sin _{\alpha}\left(\frac{(n+1) x}{2}\right)^{\alpha}, \quad \sin _{\alpha}\left(\frac{x}{2}\right)^{\alpha} \neq 0, \\
& \sum_{k=1}^{n} \cos _{\alpha}(n x)^{\alpha}=\frac{\sin _{\alpha}(n x / 2)^{\alpha}}{\sin _{\alpha}(x / 2)^{\alpha}} \cos _{\alpha}\left(\frac{(n+1) x}{2}\right)^{\alpha}, \quad \sin _{\alpha}\left(\frac{x}{2}\right)^{\alpha} \neq 0, \\
& \frac{1}{2}+\sum_{k=1}^{n} \cos _{\alpha}(n x)^{\alpha}=\frac{\sin _{\alpha}((2 n+1) x / 2)^{\alpha}}{2 \sin _{\alpha}(x / 2)^{\alpha}}, \quad \sin _{\alpha}\left(\frac{x}{2}\right)^{\alpha} \neq 0 .
\end{aligned}
$$

Remark 2.12. $i^{\alpha}$ is fractal imaginary unit, for more details, see [53-57].

\subsection{Local Fractional Fourier Series}

Definition 2.13. Suppose that $f(x) \in C_{\alpha}(-\infty, \infty)$ and $f(x)$ be $2 l$-periodic. For $k \in Z$, local fractional Fourier series of $f(x)$ is defined as, [53-55],

$$
f(x)=\frac{a_{0}}{2}+\sum_{k=1}^{\infty}\left(a_{n} \cos _{\alpha} \frac{\pi^{\alpha}(k x)^{\alpha}}{l^{\alpha}}+b_{n} \sin _{\alpha} \frac{\pi^{\alpha}(k x)^{\alpha}}{l^{\alpha}}\right),
$$

where

$$
\begin{aligned}
& a_{k}=\frac{1}{l^{\alpha}} \int_{-l}^{l} f(x) \cos _{\alpha} \frac{\pi^{\alpha}(k x)^{\alpha}}{l^{\alpha}}(d x)^{\alpha}, \\
& b_{k}=\frac{1}{l^{\alpha}} \int_{-l}^{l} f(x) \sin _{\alpha} \frac{\pi^{\alpha}(k x)^{\alpha}}{l^{\alpha}}(d x)^{\alpha}
\end{aligned}
$$

are the local fractional Fourier coefficients. 
For local fractional Fourier series (2.25), the weights of the fractional trigonometric functions are calculated as

$$
\begin{aligned}
& a_{k}=\frac{\int_{-l+t_{0}}^{l+t_{0}} f(x) \cos _{\alpha}\left(\pi^{\alpha}(k x)^{\alpha} / l^{\alpha}\right)(d x)^{\alpha}}{\int_{-l+t_{0}}^{l+t_{0}} \cos _{\alpha}^{2}\left(\pi^{\alpha}(k x)^{\alpha} / l^{\alpha}\right)(d x)^{\alpha}} \\
& a_{k}=\frac{\int_{-l+t_{0}}^{l+t_{0}} f(x) \sin _{\alpha}\left(\pi^{\alpha}(k x)^{\alpha} / l^{\alpha}\right)(d x)^{\alpha}}{\int_{-l+t_{0}}^{l+t_{0}} \sin _{\alpha}^{2}\left(\pi^{\alpha}(k x)^{\alpha} / l^{\alpha}\right)(d x)^{\alpha}} .
\end{aligned}
$$

Definition 2.14. Suppose that $f(x) \in C_{\alpha}(-\infty, \infty)$ and $f(x)$ be $2 l$-periodic. For $k \in Z$, complex generalized Mittag-Leffler form of local fractional Fourier series of $f(x)$ is defined as, [53,54],

$$
f(x)=\sum_{k=-\infty}^{\infty} C_{k} E_{\alpha}\left(\frac{\pi^{\alpha} i^{\alpha}(k x)^{\alpha}}{l^{\alpha}}\right)
$$

where the local fractional Fourier coefficients is

$$
C_{k}=\frac{1}{(2 l)^{\alpha}} \int_{-l}^{l} f(x) E_{\alpha}\left(\frac{-\pi^{\alpha} i^{\alpha}(k x)^{\alpha}}{l^{\alpha}}\right)(d x)^{\alpha} \quad \text { with } k \in Z
$$

The above generalized forms of local fractional series are valid and are also derived from the generalized Hilbert space $[53,54]$.

For local fractional Fourier series (2.28), the weights of the Mittag-Leffler functions are written in the form

$$
C_{k}=\frac{\left(1 /(2 l)^{\alpha}\right) \int_{-l+t_{0}}^{l+t_{0}} f(x) E_{\alpha}\left(-\pi^{\alpha} i^{\alpha}(k x)^{\alpha} / l^{\alpha}\right)(d x)^{\alpha}}{\left(1 /(2 l)^{\alpha}\right) \int_{-l+t_{0}}^{l+t_{0}} E_{\alpha}\left(-\pi^{\alpha} i^{\alpha}(k x)^{\alpha} / l^{\alpha}\right) \overline{E_{\alpha}\left(-\pi^{\alpha} i^{\alpha}(k x)^{\alpha} / l^{\alpha}\right)}(d x)^{\alpha}} .
$$

Above is generalized to calculate local fractional Fourier series.

\section{Solutions to Wave Equation with Fractal Vibrating String}

Now we look for particular solutions of the form

$$
u(x, t)=\phi(x) T(t)
$$

and arrive at the equations

$$
\begin{gathered}
\phi^{(2 \alpha)}+\lambda^{2 \alpha} \phi=0 \\
T^{(2 \alpha)}+a^{2 \alpha} \lambda^{2 \alpha} T=0
\end{gathered}
$$


Abstract and Applied Analysis

with the boundary conditions

$$
\phi(0)=\phi^{(\alpha)}(l)=0
$$

Equation has the solution

$$
\phi(x)=C_{1} \cos _{\alpha} \lambda^{\alpha} x^{\alpha}+C_{2} \sin _{\alpha} \lambda^{\alpha} x^{\alpha} \quad\left(C_{1}=\cos t, C_{2}=\cos t\right) .
$$

According to (3.4), for $x=0$ and $x=l$ we derive as

$$
\begin{gathered}
\phi(0)=C_{1}=0, \\
\phi(l)=\left.\phi(x)\right|_{x=l}=C_{2} \sin _{\alpha} \lambda^{\alpha} l^{\alpha}=0 .
\end{gathered}
$$

Assuming that $C_{2} \neq 0$, since otherwise $\phi(x)$ is identically zero, we find that

$$
\lambda_{n}^{\alpha} l^{\alpha}=n^{\alpha} \pi^{\alpha}
$$

where $n$ is an integer; we write

$$
\begin{gathered}
\lambda_{n}^{\alpha}=\left(\frac{n \pi}{l}\right)^{\alpha} \quad(n=0,1,2, \ldots), \\
\phi_{n}(x)=\sin _{\alpha} \lambda_{n}^{\alpha} x^{\alpha}=\sin _{\alpha} n^{\alpha}\left(\frac{\pi x}{l}\right)^{\alpha}=0 \quad(n=0,1,2, \ldots) .
\end{gathered}
$$

For $\lambda^{\alpha}=\lambda_{n}^{\alpha}$ equation (3.3) leads to

$$
T_{n}(t)=A_{n} \cos _{\alpha} a^{\alpha} \lambda_{n}^{\alpha} t^{\alpha}+B_{n} \sin _{\alpha} a^{\alpha} \lambda_{n}^{\alpha} t^{\alpha} \quad(n=0,1,2, \ldots),
$$

and therefore

$$
u_{n}(x, t)=\left(A_{n} \cos _{\alpha} a^{\alpha} \lambda_{n}^{\alpha} t^{\alpha}+B_{n} \sin _{\alpha} a^{\alpha} \lambda_{n}^{\alpha} t^{\alpha}\right) \sin _{\alpha} n^{\alpha}\left(\frac{\pi x}{l}\right)^{\alpha} \quad(n=0,1,2, \ldots) .
$$

To solve our problem, we form the local fractional Fourier series

$$
\begin{aligned}
u(x, t) & =\sum_{n=1}^{\infty} u_{n}(x, t) \\
& =\sum_{n=1}^{\infty}\left(A_{n} \cos _{\alpha} a^{\alpha} \lambda_{n}^{\alpha} t^{\alpha}+B_{n} \sin _{\alpha} a^{\alpha} \lambda_{n}^{\alpha} t^{\alpha}\right) \sin _{\alpha} n^{\alpha}\left(\frac{\pi x}{l}\right)^{\alpha},
\end{aligned}
$$


and require that

$$
\begin{aligned}
& u(x, 0)=\sum_{n=1}^{\infty} u_{n}(x, 0)=\sum_{n=1}^{\infty} A_{n} \sin _{\alpha} n\left(\frac{\pi x}{l}\right)^{\alpha}=f(x) \\
\frac{\partial^{\alpha} u(l, 0)}{\partial x^{\alpha}}= & \left.\sum_{n=1}^{\infty}\left(-A_{n} a^{\alpha} \lambda_{n}^{\alpha} \sin _{\alpha} a^{\alpha} \lambda_{n}^{\alpha} t^{\alpha}+B_{n} a^{\alpha} \lambda_{n}^{\alpha} \cos _{\alpha} a^{\alpha} \lambda_{n}^{\alpha} t^{\alpha}\right) \sin _{\alpha} n\left(\frac{\pi x}{l}\right)^{\alpha}\right|_{t=0} \\
= & \sum_{n=1}^{\infty} B_{n} a^{\alpha} \lambda_{n}^{\alpha} \sin _{\alpha} n\left(\frac{\pi x}{l}\right)^{\alpha} \\
= & g(x) .
\end{aligned}
$$

A calculation of local fractional Fourier coefficients of $f(x)$ and $g(x)$ with respect to the system $\left\{\sin _{\alpha} n^{\alpha}(\pi x / l)^{\alpha}\right\}$ is given by

$$
\begin{gathered}
A_{n}=\frac{\int_{0}^{l} f(x) \sin _{\alpha} n^{\alpha}(\pi x / l)^{\alpha}(d x)^{\alpha}}{\int_{0}^{l} \sin _{\alpha}^{2} n^{\alpha}(\pi x / l)^{\alpha}(d x)^{\alpha}} \quad(n=0,1,2, \ldots), \\
B_{n} a^{\alpha} \lambda_{n}^{\alpha}=\frac{\int_{0}^{l} g(x) \sin _{\alpha} n^{\alpha}(\pi x / l)^{\alpha}(d x)^{\alpha}}{\int_{0}^{l} \sin _{\alpha}^{2} n^{\alpha}(\pi x / l)^{\alpha}(d x)^{\alpha}} \quad(n=0,1,2, \ldots) .
\end{gathered}
$$

But $\int_{0}^{l} \sin _{\alpha}^{2} n^{\alpha}(\pi x / l)^{\alpha}(d x)^{\alpha}=l^{\alpha} / 2$ and therefore

$$
\begin{gathered}
A_{n}=\frac{2}{l^{\alpha}} \int_{0}^{l} f(x) \sin _{\alpha} \lambda_{n}^{\alpha} x^{\alpha}(d x)^{\alpha} \quad(n=0,1,2, \ldots), \\
B_{n}=\frac{2}{a^{\alpha} \lambda_{n}^{\alpha} l^{\alpha}} \int_{0}^{l} g(x) \sin _{\alpha} \lambda_{n}^{\alpha} x^{\alpha}(d x)^{\alpha} \quad(n=0,1,2, \ldots) .
\end{gathered}
$$

Thus, the solution of our problem is given by formula (3.11), where local fractional Fourier coefficients are determined. From (3.14) and (3.16), we get the harmonic vibrations

$$
u_{n}(x, t)=\left(A_{n} \cos _{\alpha} a^{\alpha} \lambda_{n}^{\alpha} t^{\alpha}+B_{n} \sin _{\alpha} a^{\alpha} \lambda_{n}^{\alpha} t^{\alpha}\right) \sin _{\alpha} \lambda_{n}^{\alpha} x^{\alpha},
$$

where

$$
\begin{gathered}
A_{n}=\frac{2}{l^{\alpha}} \int_{0}^{l} f(x) \sin _{\alpha} \lambda_{n}^{\alpha} x^{\alpha}(d x)^{\alpha} \quad(n=0,1,2, \ldots), \\
B_{n}=\frac{1}{2 / a^{\alpha} \lambda_{n}^{\alpha} l^{\alpha}} \int_{0}^{l} g(x) \sin _{\alpha} \lambda_{n}^{\alpha} x^{\alpha}(d x)^{\alpha} \quad(n=0,1,2, \ldots) .
\end{gathered}
$$


Abstract and Applied Analysis

\section{Expression Solutions with Mittag-Leffler Functions in Fractal Space}

Taking into account the relations, [57],

$$
\begin{aligned}
\sin _{\alpha} x^{\alpha} & =\frac{E_{\alpha}\left(i^{\alpha} x^{\alpha}\right)-E_{\alpha}\left(-i^{\alpha} x^{\alpha}\right)}{2 i^{\alpha}}, \\
\cos _{\alpha} x^{\alpha} & =\frac{E_{\alpha}\left(i^{\alpha} x^{\alpha}\right)+E_{\alpha}\left(-i^{\alpha} x^{\alpha}\right)}{2},
\end{aligned}
$$

we obtain the harmonic vibration with the Mittag-Leffler functions in fractal space

$$
\begin{aligned}
u_{n}(x, t)= & \left(A_{n} \frac{E_{\alpha}\left(i^{\alpha} a^{\alpha} \lambda_{n}^{\alpha} t^{\alpha}\right)+E_{\alpha}\left(-i^{\alpha} a^{\alpha} \lambda_{n}^{\alpha} t^{\alpha}\right)}{2}+B_{n} \frac{E_{\alpha}\left(i^{\alpha} a^{\alpha} \lambda_{n}^{\alpha} t^{\alpha}\right)-E_{\alpha}\left(-i^{\alpha} a^{\alpha} \lambda_{n}^{\alpha} t^{\alpha}\right)}{2 i^{\alpha}}\right) \\
& \times \frac{E_{\alpha}\left(i^{\alpha} \lambda_{n}^{\alpha} x^{\alpha}\right)-E_{\alpha}\left(-i^{\alpha} \lambda_{n}^{\alpha} x^{\alpha}\right)}{2 i^{\alpha}} \\
= & {\left[\frac{A_{n}+B_{n}}{2} E_{\alpha}\left(i^{\alpha} a^{\alpha} \lambda_{n}^{\alpha} t^{\alpha}\right)+\frac{A_{n}-B_{n}}{2} E_{\alpha}\left(-i^{\alpha} a^{\alpha} \lambda_{n}^{\alpha} t^{\alpha}\right)\right] } \\
& \times \frac{E_{\alpha}\left(i^{\alpha} \lambda_{n}^{\alpha} x^{\alpha}\right)-E_{\alpha}\left(-i^{\alpha} \lambda_{n}^{\alpha} x^{\alpha}\right)}{2 i^{\alpha}} \\
= & \frac{A_{n}+B_{n}}{4 i^{\alpha}}\left\{E_{\alpha}\left[i^{\alpha} \lambda_{n}^{\alpha}(a t+x)^{\alpha}\right]-E_{\alpha}\left[i^{\alpha} \lambda_{n}^{\alpha}(a t-x)^{\alpha}\right]\right\} \\
& +\frac{A_{n}-B_{n}}{4 i^{\alpha}}\left\{E_{\alpha}\left[i^{\alpha} \lambda_{n}^{\alpha}(x-a t)^{\alpha}\right]-E_{\alpha}\left[-i^{\alpha} \lambda_{n}^{\alpha}(a t+x)^{\alpha}\right]\right\},
\end{aligned}
$$

where its coefficients are

$$
\begin{aligned}
A_{n} & =\frac{2}{l^{\alpha}} \int_{0}^{l} f(x) \sin _{\alpha} \lambda_{n}^{\alpha} x^{\alpha}(d x)^{\alpha} \\
& =\frac{2}{l^{\alpha}} \int_{0}^{l} f(x) \frac{E_{\alpha}\left(i^{\alpha} \lambda_{n}^{\alpha} x^{\alpha}\right)-E_{\alpha}\left(-i^{\alpha} \lambda_{n}^{\alpha} x^{\alpha}\right)}{2 i^{\alpha}}(d x)^{\alpha} \\
& =\frac{1}{i^{\alpha} l^{\alpha}} \int_{0}^{l} f(x)\left(E_{\alpha}\left(i^{\alpha} \lambda_{n}^{\alpha} x^{\alpha}\right)-E_{\alpha}\left(-i^{\alpha} \lambda_{n}^{\alpha} x^{\alpha}\right)\right)(d x)^{\alpha} \quad(n=0,1,2, \ldots), \\
B_{n} & =\frac{2}{a^{\alpha} \lambda_{n}^{\alpha} l^{\alpha}} \int_{0}^{l} g(x) \sin _{\alpha} \lambda_{n}^{\alpha} x^{\alpha}(d x)^{\alpha} \\
& =\frac{2}{a^{\alpha} \lambda_{n}^{\alpha} l^{\alpha}} \int_{0}^{l} g(x) \frac{E_{\alpha}\left(i^{\alpha} \lambda_{n}^{\alpha} x^{\alpha}\right)-E_{\alpha}\left(-i^{\alpha} \lambda_{n}^{\alpha} x^{\alpha}\right)}{2 i^{\alpha}}(d x)^{\alpha} \\
& =\frac{1}{a^{\alpha} \lambda_{n}^{\alpha} l^{\alpha} i^{\alpha}} \int_{0}^{l} g(x)\left(E_{\alpha}\left(i^{\alpha} \lambda_{n}^{\alpha} x^{\alpha}\right)-E_{\alpha}\left(-i^{\alpha} \lambda_{n}^{\alpha} x^{\alpha}\right)\right)(d x)^{\alpha} \quad(n=0,1,2, \ldots) .
\end{aligned}
$$


Hereby, we always find that

$$
\left|f(x)-f\left(x_{0}\right)\right|<\varepsilon^{\alpha}, \quad\left|g(x)-g\left(x_{0}\right)\right|<\varepsilon^{\alpha}
$$

with $\left|x-x_{0}\right|<\delta$, for $\varepsilon, \delta>0$ and $\varepsilon, \delta \in R$.

Hence the boundary conditions are fractal and solution with Mittag-Leffler functions in fractal space is given by

$$
\begin{aligned}
u(x, t)= & \sum_{n=1}^{\infty} u_{n}(x, t) \\
= & \sum_{n=1}^{\infty} \frac{A_{n}+B_{n}}{4 i^{\alpha}}\left\{E_{\alpha}\left[i^{\alpha} \lambda_{n}^{\alpha}(a t+x)^{\alpha}\right]-E_{\alpha}\left[i^{\alpha} \lambda_{n}^{\alpha}(a t-x)^{\alpha}\right]\right\} \\
& +\sum_{n=1}^{\infty} \frac{A_{n}-B_{n}}{4 i^{\alpha}}\left\{E_{\alpha}\left[i^{\alpha} \lambda_{n}^{\alpha}(x-a t)^{\alpha}\right]-E_{\alpha}\left[-i^{\alpha} \lambda_{n}^{\alpha}(a t+x)^{\alpha}\right]\right\},
\end{aligned}
$$

where its coefficients are derived as

$$
\begin{gathered}
A_{n}=\frac{1}{i^{\alpha} l^{\alpha}} \int_{0}^{l} f(x)\left(E_{\alpha}\left(i^{\alpha} \lambda_{n}^{\alpha} x^{\alpha}\right)-E_{\alpha}\left(-i^{\alpha} \lambda_{n}^{\alpha} x^{\alpha}\right)\right)(d x)^{\alpha} \quad(n=0,1,2, \ldots), \\
B_{n}=\frac{1}{a^{\alpha} \lambda_{n}^{\alpha} l^{\alpha} i^{\alpha}} \int_{0}^{l} g(x)\left(E_{\alpha}\left(i^{\alpha} \lambda_{n}^{\alpha} x^{\alpha}\right)-E_{\alpha}\left(-i^{\alpha} \lambda_{n}^{\alpha} x^{\alpha}\right)\right)(d x)^{\alpha} \quad(n=0,1,2, \ldots) .
\end{gathered}
$$

\section{Conclusions}

We applied the technique of the local fractional Fourier series to treat with the local fractional wave equation in fractal vibrating string. When contrasted with other analytical methods, such as the heat-balance integral method, the homotopy perturbation method [11], the variational iteration method [29], the exp-function method [58], the fractional variational iteration method [31, 32], the fractional complex method [33-37], and others [59-61], the present method combines the following two advantages. The boundary conditions to the governing equations are local fractional continuous (the functions are nondifferential functions in fractal space) because we employ the local fractional Fourier series, derived from local fractional calculus, to deal with them. The governing equations with fractal behaviors in media are structured based on the local fractional calculus. The way plays a crucial role in local fractional calculus. This technique is efficient for the applied scientists to process these differential equations with the local fractional differential operators in fractal space. This paper that is an outstanding example of application of local fractional Fourier series to the local fractional differential operators is given to elucidate the solution processes and reliable results. 


\section{Acknowledgments}

This paper is sponsored by the National Natural Science Foundation of China (NSFC, Grant U1204703), the Key Scientific and Technological Project of Henan Province (122102310004), the Fundamental Research Funds for the Central Universities (HUST: 2012QN087, 2012QN088), and the Innovation Scientists and Technicians Troop Construction Projects of Zhengzhou City (10LJRC190, 121PRKXF658-4).

\section{References}

[1] R. Hilfe, Applications of Fractional Calculus in Physics, World Scientific, River Edge, NJ, USA, 2000.

[2] J. Sabatier, O. P. Agrawal, and J. A. Tenreiro, Machado, Advances in Fractional Calculus: Theoretical Developments and Applications in Physics and Engineering, Springer, New York, NY, USA, 2007.

[3] K. B. Oldham and J. Spanier, The Fractional Calculus, Academic Press, London, UK, 1974.

[4] K. S. Miller and B. Ross, An Introduction To the Fractional Calculus and Fractional Differential Equations, John Wiley \& Sons, New York, NY, USA, 1993.

[5] I. Podlubny, Fractional Differential Equations, vol. 198, Academic Press, San Diego, Calif, USA, 1999.

[6] S. G. Samko, A. A. Kilbas, and O. I. Marichev, Fractional Integrals and Derivatives, Gordon and Breach Science Publishers, Yverdon, Switzerland, 1993.

[7] A. A. Kilbas, H. M. Srivastava, and J. J. Trujillo, Theory and Applications of Fractional Differential Equations, vol. 204, Elsevier, Amsterdam, The Netherlands, 2006.

[8] W. R. Schneider and W. Wyss, "Fractional diffusion and wave equations," Journal of Mathematical Physics, vol. 30, no. 1, pp. 134-144, 1989.

[9] O. P. Agrawal, "Solution for a fractional diffusion-wave equation defined in a bounded domain," Nonlinear Dynamics, vol. 29, no. 1-4, pp. 145-155, 2002.

[10] A. M. A. El-Sayed, "Fractional-order diffusion-wave equation," International Journal of Theoretical Physics, vol. 35, no. 2, pp. 311-322, 1996.

[11] H. Jafari and S. Seifi, "Homotopy analysis method for solving linear and nonlinear fractional diffusion-wave equation," Communications in Nonlinear Science and Numerical Simulation, vol. 14, no. 5, pp. 2006-2012, 2009.

[12] Y. Povstenko, "Non-axisymmetric solutions to time-fractional diffusion-wave equation in an infinite cylinder," Fractional Calculus and Applied Analysis, vol. 14, no. 3, pp. 418-435, 2011.

[13] F. Mainardi and G. Pagnini, "The Wright functions as solutions of the time-fractional diffusion equation," Applied Mathematics and Computation, vol. 141, no. 1, pp. 51-62, 2003.

[14] Y. Luchko, "Some uniqueness and existence results for the initial-boundary-value problems for the generalized time-fractional diffusion equation," Computers $\mathcal{E}$ Mathematics with Applications, vol. 59, no. 5, pp. 1766-1772, 2010.

[15] M. M. Meerschaert, D. A. Benson, H.-P. Scheffler, and B. Baeumer, "Stochastic solution of space-time fractional diffusion equations," Physical Review E, vol. 65, no. 4, Article ID 041103, 4 pages, 2002.

[16] S. D. Eidelman and A. N. Kochubei, "Cauchy problem for fractional diffusion equations," Journal of Differential Equations, vol. 199, no. 2, pp. 211-255, 2004.

[17] T. Sandev and Ž. Tomovski, "The general time fractional wave equation for a vibrating string," Journal of Physics A, vol. 43, no. 5, Article ID 055204, 2010.

[18] T. M. Atanackovic and B. Stankovic, "Generalized wave equation in nonlocal elasticity," Acta Mechanica, vol. 208, no. 1-2, pp. 1-10, 2009.

[19] J. Dong and M. Xu, "Space-time fractional Schrödinger equation with time-independent potentials," Journal of Mathematical Analysis and Applications, vol. 344, no. 2, pp. 1005-1017, 2008.

[20] S. Z. Rida, H. M. El-Sherbiny, and A. A. M. Arafa, "On the solution of the fractional nonlinear Schrödinger equation," Physics Letters A, vol. 372, no. 5, pp. 553-558, 2008.

[21] R. Scherer, S. L. Kalla, L. Boyadjiev, and B. Al-Saqabi, "Numerical treatment of fractional heat equations," Applied Numerical Mathematics, vol. 58, no. 8, pp. 1212-1223, 2008.

[22] S. Momani, "An explicit and numerical solutions of the fractional KdV equation," Mathematics and Computers in Simulation, vol. 70, no. 2, pp. 110-118, 2005.

[23] R. Metzler and T. F. Nonnenmacher, "Space- and time-fractional diffusion and wave equations, fractional Fokker-Planck equations, and physical motivation," Chemical Physics, vol. 84, pp. 67-90, 2002.

[24] P. Paradisi, R. Cesari, F. Mainardi, and F. Tampieri, "The fractional Fick's law for non-local transport processes," Physica A, vol. 293, no. 1-2, pp. 130-142, 2001. 
[25] Y. Zhou and F. Jiao, "Nonlocal Cauchy problem for fractional evolution equations," Nonlinear Analysis, vol. 11, no. 5, pp. 4465-4475, 2010.

[26] V. E. Tarasov, "Fractional Heisenberg equation," Physics Letters A, vol. 372, no. 17, pp. 2984-2988, 2008.

[27] V. E. Tarasov and G. M. Zaslavsky, "Fractional Ginzburg-Landau equation for fractal media," Physica A, vol. 354, no. 15, pp. 249-261, 2005.

[28] V. E. Tarasov, "Fractional hydrodynamic equations for fractal media," Annals of Physics, vol. 318, no. 2, pp. 286-307, 2005.

[29] J.-H. He, "Approximate analytical solution for seepage flow with fractional derivatives in porous media," Computer Methods in Applied Mechanics and Engineering, vol. 167, no. 1-2, pp. 57-68, 1998.

[30] Q. Wang, "Numerical solutions for fractional KdV-Burgers equation by Adomian decomposition method," Applied Mathematics and Computation, vol. 182, no. 2, pp. 1048-1055, 2006.

[31] G. C. Wu and E. W. M. Lee, "Fractional variational iteration method and its application," Physics Letters A, vol. 374, no. 25, pp. 2506-2509, 2010.

[32] J.-H. He, "A short remark on fractional variational iteration method," Physics Letters A, vol. 375, no. 38, pp. 3362-3364, 2011.

[33] Z.-B. Li and J.-H. He, "Fractional complex transform for fractional differential equations," Mathematical \& Computational Applications, vol. 15, no. 5, pp. 970-973, 2010.

[34] J.-H. He, S. K. Elagan, and Z. B. Li, "Geometrical explanation of the fractional complex transform and derivative chain rule for fractional calculus," Physics Letters A, vol. 376, no. 4, pp. 257-259, 2012.

[35] Z. B. Li and J. H. He, "Application of the fractional complex transform to fractional differential equations," Nonlinear Science Letters A, vol. 2, no. 3, pp. 121-126, 2011.

[36] Z. B. Li, W. H. Zhu, and J. H. He, "Exact solutions of time-fractional heat conduction equation by the fractional complex transform," Thermal Science, vol. 16, no. 2, pp. 335-338, 2012.

[37] Q. L. Wang, J. H. He, and Z. B. Li, "Fractional model for heat conduction in polar bear hairs," Thermal Science, vol. 16, no. 2, pp. 339-342, 2012.

[38] G. Jumarie, "On the representation of fractional Brownian motion as an integral with respect to (dt) ${ }^{a}$," Applied Mathematics Letters, vol. 18, no. 7, pp. 739-748, 2005.

[39] G. Jumarie, "Probability calculus of fractional order and fractional Taylor's series application to Fokker-Planck equation and information of non-random functions," Chaos, Solitons and Fractals, vol. 40, no. 3, pp. 1428-1448, 2009.

[40] G. Jumarie, "Laplace's transform of fractional order via the Mittag-Leffler function and modified Riemann-Liouville derivative," Applied Mathematics Letters, vol. 22, no. 11, pp. 1659-1664, 2009.

[41] G.-C. Wu, "Adomian decomposition method for non-smooth initial value problems," Mathematical and Computer Modelling, vol. 54, no. 9-10, pp. 2104-2108, 2011.

[42] K. M. Kolwankar and A. D. Gangal, “Local fractional Fokker-Planck equation,” Physical Review Letters, vol. 80, no. 2, pp. 214-217, 1998.

[43] A. Carpinter and A. Sapora, "Diffusion problems in fractal media defined on Cantor sets," ZAMM Journal of Applied Mathematics and Mechanics, vol. 90, no. 3, pp. 203-210, 2010.

[44] K. M. Kolwankar and A. D. Gangal, "Fractional differentiability of nowhere differentiable functions and dimensions," Chaos, vol. 6, no. 4, pp. 505-513, 1996.

[45] X. R. Li, Fractional calculus, fractal geometry, and stochastic processes [Ph.D. thesis], University of Western Ontario, Ontario, Canada, 2003.

[46] A. Babakhani and V. D. Gejji, “On calculus of local fractional derivatives," Journal of Mathematical Analysis and Applications, vol. 270, no. 1, pp. 66-79, 2002.

[47] A. Parvate and A. D. Gangal, "Calculus on fractal subsets of real line. I. Formulation," Fractals, vol. 17 , no. 1 , pp. 53-81, 2009.

[48] F. Ben Adda and J. Cresson, "About non-differentiable functions," Journal of Mathematical Analysis and Applications, vol. 263, no. 2, pp. 721-737, 2001.

[49] A. Carpinteri, B. Chiaia, and P. Cornetti, "The elastic problem for fractal media: basic theory and finite element formulation," Computers E Structures, vol. 82, no. 6, pp. 499-508, 2004.

[50] A. Carpinteri and P. Cornetti, "A fractional calculus approach to the description of stress and strain localization in fractal media," Chaos, Solitons, Fractals, vol. 13, no. 1, pp. 85-94, 2002.

[51] Y. Chen, Y. Yan, and K. Zhang, "On the local fractional derivative," Journal of Mathematical Analysis and Applications, vol. 362, no. 1, pp. 17-33, 2010.

[52] A. Carpinteri, B. Chiaia, and P. Cornetti, "On the mechanics of quasi-brittle materials with a fractal microstructure," Engineering Fracture Mechanics, vol. 70, no. 6, pp. 2321-2349, 2003. 
[53] X. J. Yang, "Local fractional integral transforms," Progress in Nonlinear Science, vol. 4, pp. 1-225, 2011.

[54] X. J. Yang, Local Fractional Functional Analysis and Its Applications, Asian Academic Publisher, Hong Kong, 2011.

[55] X. J. Yang, "Local fractional calculus and its applications," in Proceedings of the 5th IFAC Workshop Fractional Differentiation and Its Applications, (FDA '12), pp. 1-8, Nanjing, China, 2012.

[56] X. J. Yang, M. K. Liao, and J. W. Chen, "A novel approach to processing fractal signals using the Yang-Fourier transforms," Procedia Engineering, vol. 29, pp. 2950-2954, 2012.

[57] X. J. Yang, Advanced Local Fractional Calculus and Its Applications, World Science Publisher, New York, NY, USA, 2012.

[58] S. Zhang and H.-Q. Zhang, "Fractional sub-equation method and its applications to nonlinear fractional PDEs," Physics Letters A, vol. 375, no. 7, pp. 1069-1073, 2011.

[59] J. H. He, "Analytical methods for thermal science-an elementary introduction," Thermal Science, vol. 15, pp. S1-S3, 2011.

[60] J. H. He, “A new fractal derivation," Thermal Science, vol. 15, pp. S145-S147, 2011.

[61] J. H. He, "Asymptotic methods for solitary solutions and compactons," Abstract and Applied Analysis, vol. 2012, Article ID 916793, 130 pages, 2012. 


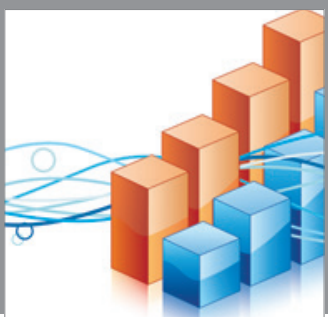

Advances in

Operations Research

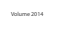

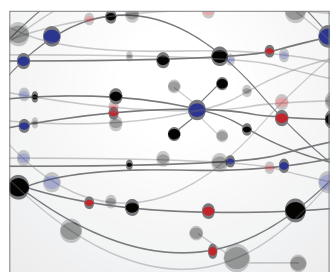

\section{The Scientific} World Journal
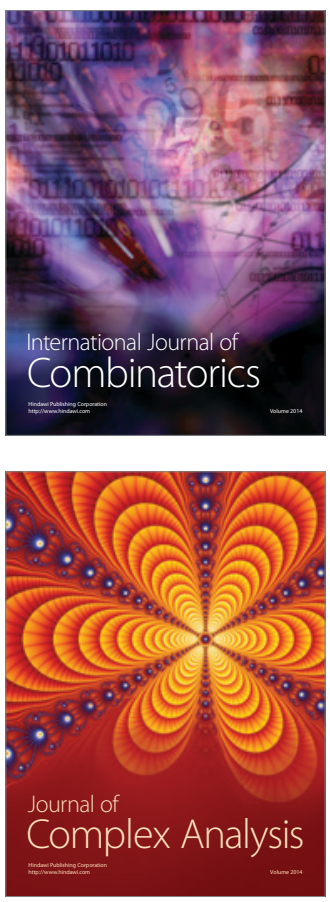

International Journal of

Mathematics and

Mathematical

Sciences
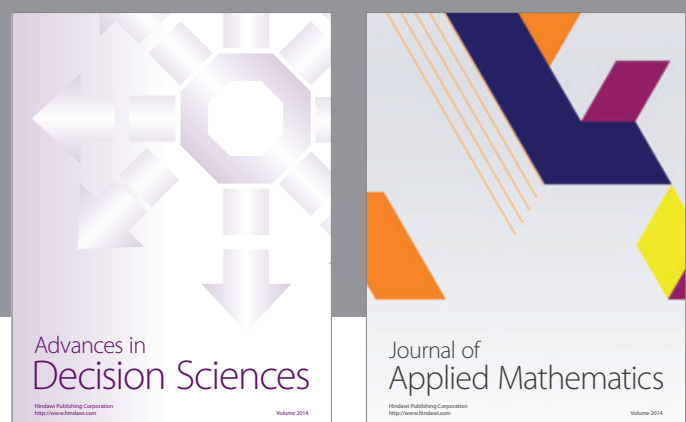

Journal of

Applied Mathematics
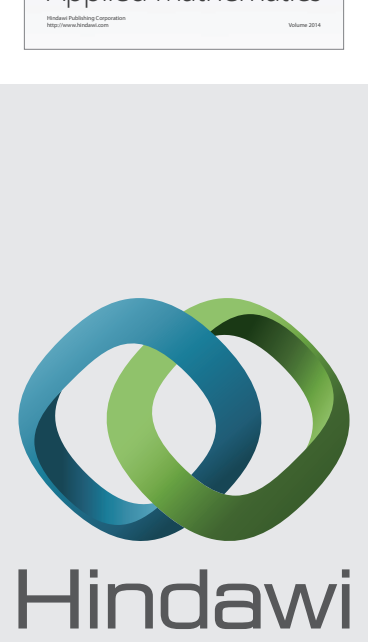

Submit your manuscripts at http://www.hindawi.com
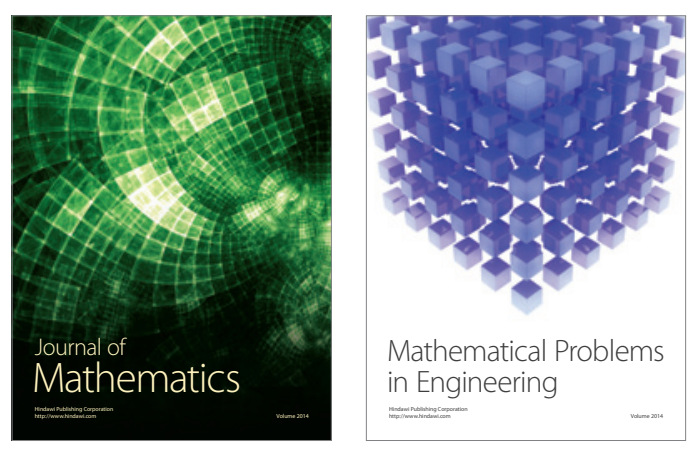

Mathematical Problems in Engineering
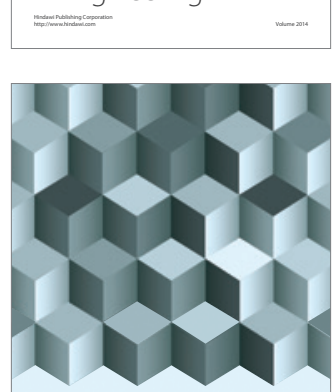

Journal of

Function Spaces
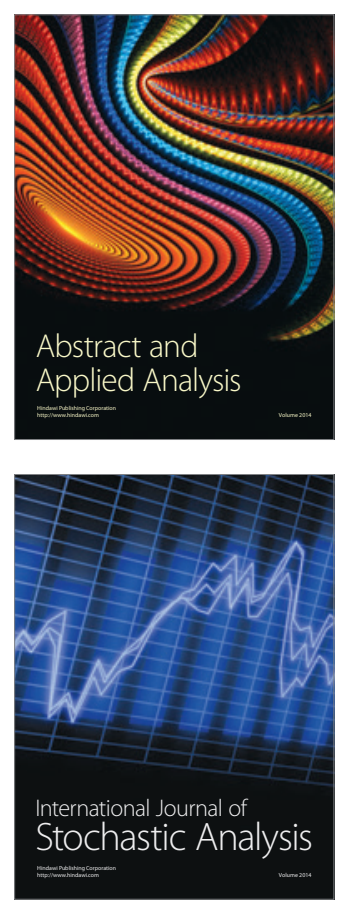

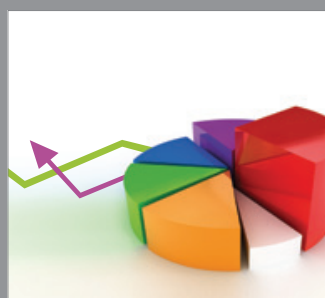

ournal of

Probability and Statistics

Promensencen
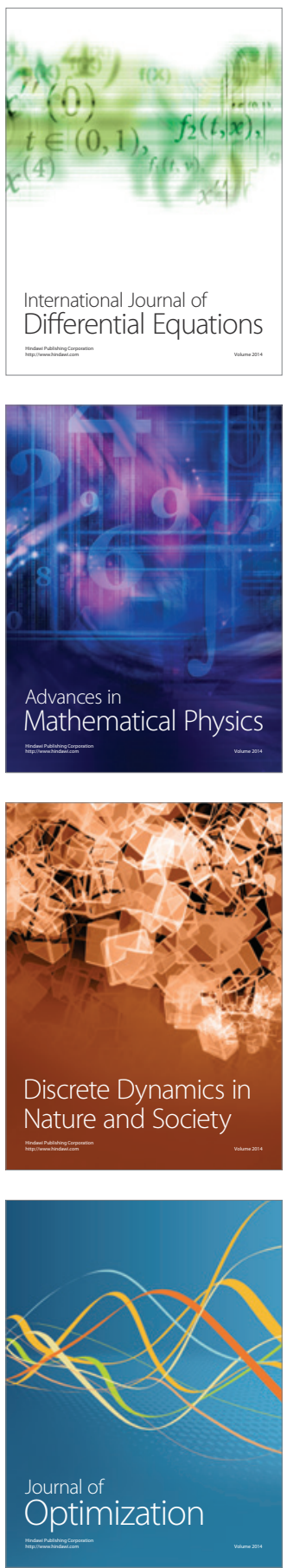